WI - Innovatives Produkt

\title{
Produkte zum IT-gestützten Social Networking in Unternehmen
}

\section{Die Autoren}

\section{Michael Koch Alexander Richter Andreas Schlosser}

Prof. Dr. Michael Koch Alexander Richter Andreas Schlosser Universität der Bundeswehr München Werner-Heisenberg-Weg 39 85577 Neubiberg \{michael.koch I a.richter I andreas.schlosser\}@unibw.de

Eingereicht am 2007-06-28, nach einer Überarbeitung angenommen am 2007-09-03 durch Prof. Dr. Spann. nahmen (wie von Youtube.com) oder Börsengängen (wie durch Xing.de) mancher erst seit wenigen Monaten existierenden Unternehmen [Alby06, 5].

Doch nicht nur für die private Nutzung sind Web 2.0 Dienste interessant. Auch für IT-Verantwortliche von Unternehmen und die Wirtschaftsinformatik im Allgemeinen kann die Betrachtung der Systeme und ihrer Charakteristika neue Einsichten zur Unterstützung von Zusammenarbeit und Wissensmanagement in Unternehmen erschließen.

Ein gemeinsamer zentraler Punkt aller Web-2.0-Dienste ist die Nutzerzentriertheit, die es allen Nutzern ermöglicht, sich aktiv an der Erstellung der Inhalte (Daten, Meta-Daten, Kommentare) zu beteiligen. Um eine möglichst große freiwillige Nutzerbeteiligung $\mathrm{zu}$ erreichen versucht man es den Benutzern möglichst einfach zu machen, etwas beizutragen, z. B. durch eine Bereitstellung der Dienste als Web-Anwendungen und die besondere Berücksichtigung der einfachen Benutzbarkeit der
Dienste und deren Kombinierbarkeit. In diesem Zusammenhang wird ein weiteres Charakteristikum von Web-2.0-Diensten deutlich: Man versucht nicht, die Benutzer zu Communitys zu bündeln und die Motivation zur Inhaltserstellung auf Gruppeninteressen fußen zu lassen („We“-Mentalität). Der Fokus liegt stattdessen auf dem Nutzen für den einzelnen Benutzer und der intrinsischen Motivation der einzelnen Nutzer („Me“-Mentalität). Um all das zu erreichen, kommen neue Techniken und Konzepte wie z. B. Ajax, RSS oder Mashups zum Einsatz.

\subsection{Social Software und Enterprise 2.0}

Eine Gruppe von Anwendungen im Web 2.0 lässt sich unter dem Begriff „Social Software" zusammenfassen. Unter Social Software werden dabei Anwendungssysteme verstanden, die auf Basis neuer Entwicklungen im Bereich der Internettech-

\section{Einleitung}

\subsection{Web 2.0}

Obwohl bereits seit 2005 weltweit benutzt [ORei05], erlangte der Begriff „Web 2.0“ in Deutschland gerade Anfang 2007 eine große Aufmerksamkeit und schaffte in verschiedenen Kontexten den Sprung auf die Titelseiten nahezu aller Massenprintmedien. Die Ursache für die anhaltende Begeisterung ist v. a. in den beachtlichen Benutzerzuwächsen zahlreicher Dienste und den daraus erwachsenden Folgen zu suchen, z. B. multimillionenschwere Über-

\section{Kernpunkte}

Der Beitrag beschäftigt sich mit der Verwendung von Social-Networking-Diensten (Social Networking Services) im Unternehmenskontext.

- Social-Networking-Dienste unterstützen Unternehmen dabei, ihr Humankapital besser auszuschöpfen.

- Social-Networking-Dienste können ein zentrales Element der Expertensuche im Unternehmen sein.

- Neben offenen Social-Networking-Diensten entwickelt sich ein Markt für Software zum Aufbau geschlossener, nur im Intranet zugreifbarer und unternehmensindividuell anpassbarer und integrierbarer Social-Networking-Dienste.

Stichworte: Social-Networking-Dienst, Expertensuche, Expertenverzeichnis, Wissensmanagement, Web 2.0, Social Software, Enterprise 2.0 
nologien und unter Ausnutzung von Netzwerk- und Skaleneffekten indirekte und direkte zwischenmenschliche Interaktion (Koexistenz, Kommunikation, Koordination, Kooperation) auf breiter Basis ermöglichen und die Beziehungen ihrer Nutzer im World Wide Web abbilden und unterstützen [RiKo07, 5].

Neben den bereits oben angesprochenen Eigenschaften von Web-2.0-Anwendungen, die sich auch auf Social Software übertragen lassen, stellen Hippner und Wilde [HiWi05, 1] heraus, dass die Grundidee dieser Art von Diensten in der Selbstorganisation der Benutzer liegt. Lediglich eine Handvoll von Konventionen regelt das gemeinsame Handeln auf den Plattformen im WWW. Dies trägt maßgeblich dazu bei, die Nutzung der Dienste so einfach wie möglich zu machen und damit möglichst großen Nutzen (für den Einzelnen und die Gruppe) zu generieren.

Für den Einsatz von Social Software im (internen) Unternehmenskontext wurde von Andrew McAfee der Begriff „Enterprise 2.0" geprägt [McAf06]. Neben Weblogs, Wikis und Social Tagging (-Diensten) stellen Social-Networking-Dienste einen wichtigen Teilbereich von Enterprise 2.0 dar - sie decken vor allem die Bereiche des Beziehungs- und Identitätsmanagements ab. Laut einer weltweiten Umfrage von McKinsey werden Social-NetworkingDienste derzeit in großen Unternehmen sogar häufiger eingesetzt $(37 \%)$ als Wikis (33\%) oder Weblogs (32\%) [BuMa07, 3].

Im Folgenden wird herausgearbeitet, wie Social-Networking-Dienste allgemein die (Zusammen-)Arbeit in einem Unternehmen verändern und die Mitarbeiter unterstützen können (Abschnitt 2). Anschließend wird ein Überblick über konkrete Lösungen aus diesem Bereich gegeben (Abschnitt 3) sowie ein Ausblick au zukünftige Entwicklungen gewagt (Abschnitt 4).

\section{Nutzen und IT-Unter- stützung sozialer Netzwerke}

\subsection{Soziale Netzwerke}

Schon in der Diskussion rund um rechnergestützte Gruppenarbeit (Computer-Supported Cooperative Work, CSCW) wurde angemerkt, dass die Betrachtung der unterstützten Individuen und ihrer sozialen Netzwerke wichtiger sei als die Betrachtung der Gruppen und Teams [Well97; Well01; NaWSO2].

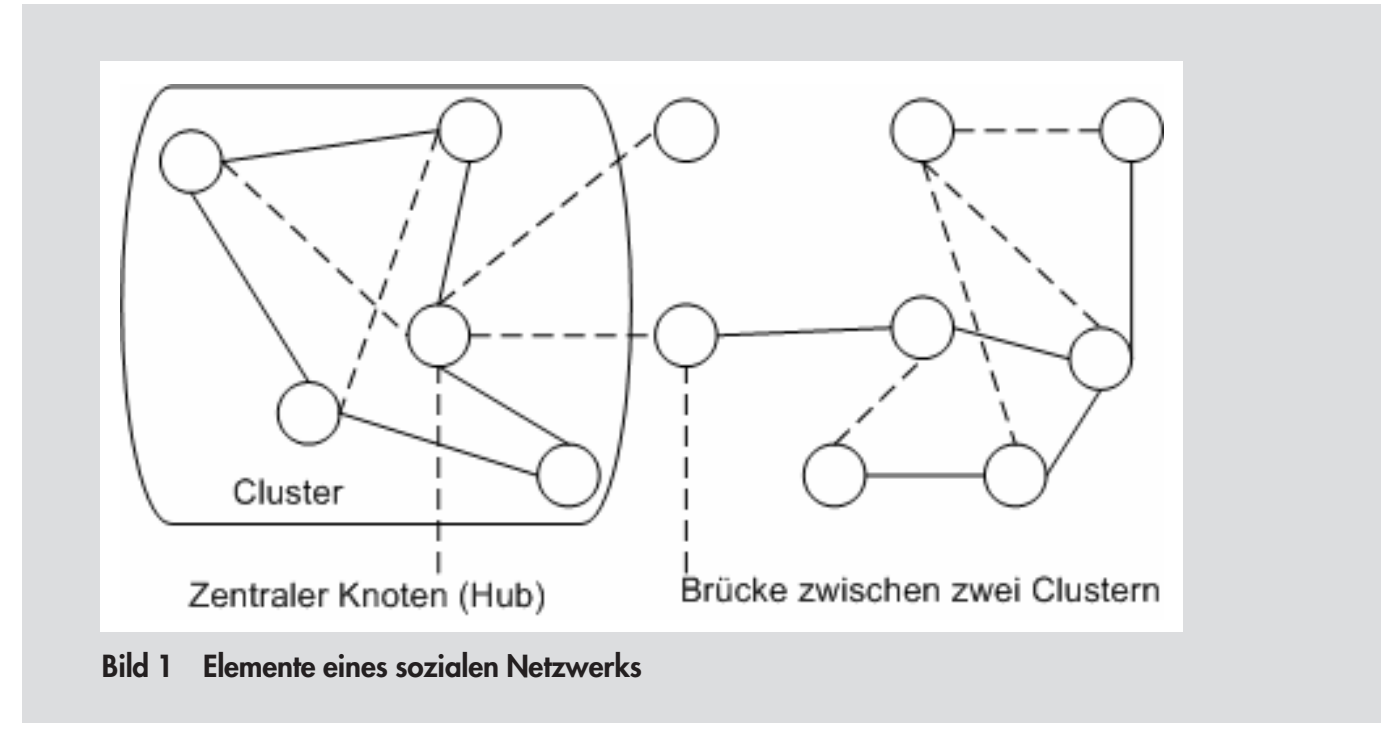

Unter einem sozialen Netzwerk versteht man dabei allgemein eine abgegrenzte Menge von Knoten in der Form von Akteuren oder Akteursgruppen und einer Menge von Kanten zwischen diesen Knoten. Die Kanten beschreiben soziale Interaktionen oder Beziehungen (z. B. Kommunikationsbeziehungen oder Bekanntschaften) zwischen den Akteuren. D. h. in einem sozialen Netzwerk kann abgebildet sein, wer mit wem kommuniziert, wer wen kennt oder wem vertraut. Bild 1 zeigt ein solches Netzwerk mit zwei Typen von Interaktionen oder Beziehungen. Bei der Erhebung und Visualisierung von sozialen Netzwerken werden neben den Verbindungen der Akteure im Allgemeinen insbesondere Cluster (Gruppen von sich nahe stehenden Personen) und Hubs (Personen, die besonders viele Kontakte $\mathrm{zu}$ anderen haben) sichtbar.

Obwohl soziale Netzwerke gerade in den letzten Jahren in den Fokus verschiedener Forschungsrichtungen rücken (u. a. auch als Folge der zunehmend vernetzten und sich vernetzenden Gesellschaft [Cast03, 1 ff.]) liegen mehrere bemerkenswerte Erkenntnisse der Netzwerkforschung schon Jahrzehnte zurück.

Mitte der 1930er führte Jacob Moreno [More34] mehrere bis heute viel zitierte soziometrische Analysen durch. Dabei handelt es sich um direkte Vorläufer des heute als Analyse sozialer Netzwerke (Social Networking Analysis, SNA) zunehmend bekannteren Ansatzes. Eine SNA untersucht die Struktur sozialer Beziehungen innerhalb einer Gruppe um informelle Verbindungen zwischen den Akteuren aufzuzeigen [EhCa05, 1]. In einem Unternehmen stände eine SNA also vor folgen- der Fragestellung: Wer (die Akteure einer Gruppe) arbeitet wie (Art der Kommunikation) unter welchen Voraussetzungen (welche Anreize existieren etc.) zusammen?

Mitte der 1960er fand der Psychologe Stanley Milgram [Milg67] Anhaltspunkte dafür, dass jeder Mensch auf der Welt mit jedem anderen über eine überraschend kurze Kette von Bekanntschaftsbeziehungen verbunden ist. In diesem Zusammenhang ist auch vom „small world phenomenon“ oder von "6-degrees-of-separation“ die Rede, d. h., dass jeder jeden über sechs Ecken kennt. Diese Eigenschaft ist darauf zurück zu führen, dass es sich bei sozialen Netzwerken normalerweise um skalenfreie Netzwerke handelt, also die Anzahl der Verbindungen nicht gleich über alle Knoten verteilt ist, sondern viele wenig vernetzte Knoten und einige stark vernetzte Hubs existieren [siehe hierzu auch HeSK06; BaBo03; Glad00].

Die Auswirkungen der sozialen Vernetzung im Arbeitskontext untersuchte Anfang der 1970er der Soziologe Mark Granovetter [Gran73] und fasste seine Ergebnisse in der Theorie der „strength of weak ties" zusammen. Diese besagt, dass gerade Verbindungen $\mathrm{zu}$ flüchtigen Bekannten oftmals zu großen Informationsvorsprüngen führen, weil diese vermehrt Kontakt $\mathrm{zu}$ anderen Clustern pflegen. Seine Begründung dafür: Es besteht eine größere Wahrscheinlichkeit, dass schwache Verbindungen („weak ties") in anderen Kreisen verkehren (und damit Zugang zu anderen Informationen haben) als eng befreundete Kontakte. Die „weak ties" sind also in Wirklichkeit „strong ties“. 


\subsection{Networking}

Bezogen auf die Herausforderungen in Unternehmen zeigt sich eine hohe Relevanz der oben genannten Erkenntnisse aus der Sozialforschung. Dabei steht das Networking im Vordergrund. Man versteht darunter den Aufbau von Beziehungen innerhalb von und zwischen Gruppen [Furn97, 541]. Durch diesen Beziehungsaufbau finden Akteure mit gleichen Interessen und Themengebieten zueinander und können sich in vielfältigsten Formen austauschen und eine Zusammenarbeit anstreben.

Auch Scheler [Sche00, 26] sieht Networking als eine methodische und systematische Tätigkeit, deren Sinn darin besteht, Kontakte $\mathrm{zu}$ Menschen $\mathrm{zu}$ suchen und Beziehungen zu diesen zu pflegen.

Für das Individuum bedeutet Networking eine Investition in Kontakte mit anderen Individuen, um später, wenn die Beziehung benötigt wird, Suchkosten und Kosten für den Aufbau eines gemeinsamen Kontextes zu minimieren. Ein ähnliches Ziel verfolgen Unternehmen, die das Networking ihrer Mitarbeiter aktiv unterstützen: Die individuellen Kosten für die einzelnen Mitarbeiter sollen weitestmöglich gesenkt werden, um die Vernetzung der Mitarbeiter zu stärken. Dieser Aufbau von strong ties und weak ties hat für Unternehmen den Vorteil, dass Mitarbeiter leichter Kontakt zu Kollegen, die sich mit ähnlichen Problemen befassen, finden. Auf diese Weise wird redundante Arbeit verringert und der Austausch von Erfahrungswissen erleichtert.

Bisher wurden in Unternehmen häufig sogenannte Communities of Practice [Weng98] gefördert, um den persönlichen Wissensaustausch der Mitarbeiter zu unterstützen. Während Communitys jedoch eher auf strong ties setzen, verfolgt die explizite Unterstützung sozialer Netzwerke das gleiche Ziel, will dabei jedoch zusätzlich die weak ties nutzen. So bilden die Beteiligten in Communitys eine Einheit mit klarer Abgrenzung nach außen. In Netzwerken existieren zwar Cluster, aber keine nach außen abgegrenzten Einheiten.

Netzwerke entsprechen der Idee, dass die Individuen getrennt voneinander agieren und je nach Bedarf bereits vorhandene Kanten nutzen oder neue Kanten zu bisher unbekannten Knoten ausbilden. Durch den Fokus auf die Eigeninteressen und den Verzicht der Unterordnung unter Gruppeninteressen entsteht bei den Mitarbeitern eine höhere Motivation zur Beteiligung. Eine ausführliche Diskussion zu verschiedenen Typen von Netzwerken in Unter- nehmen und zu deren Nutzen nehmen beispielsweise Cross et al. [CrLW05] vor.

Es ist wichtig festzuhalten, dass Communitys und Netzwerke keinen Gegensatz darstellen. So bilden sich häufig Communitys bzw. Gruppen innerhalb großer sozialer Netzwerke, und genauso sind Netzwerke innerhalb größerer Communitys vorhanden. Die Frage ist also nicht, was in einem sozialen System existiert - Netzwerke oder Communitys - sondern was man hauptsächlich unterstützen will.

\subsection{Social-Networking-Dienste}

Die technologischen und technischen Entwicklungen der letzten Jahre machen es möglich, soziale Netzwerke digital abzubilden und erlauben es Menschen darüber hinaus sehr einfach Kontakt miteinander aufzunehmen und diesen aufrecht zu erhalten. Als Social-Networking-Dienste bezeichnen wir Dienste, die ihren Nutzern Funktionalitäten zum Identitätsmanagement (d. h. zur Darstellung der eigenen Person i. d. R. in Form eines Profils) zur Verfügung stellen und darüber hinaus die Vernetzung mit anderen Nutzern (und so die Verwaltung eigener Kontakte und Pflege des Netzwerks) ermöglichen. Social-Networking-Dienste stellen dabei eine Klasse der in Abschnitt 1 angesprochenen Social Software dar.

Die Grundfunktionen solcher SocialNetworking-Dienste sind:

- Identitätsmanagement: Möglichkeiten zur Eingabe, Pflege und Darstellung von Aspekten der eigenen Person,

- Beziehungsmanagement: Möglichkeiten zur Verwaltung der eigenen Kontakte und Pflege des Netzwerkes,

- Visualisierung von Profilen und Netzwerken (als Graph, Menge von Verbindungspfaden, Tag Cloud).

Zusätzlich zu den Grundfunktionalitäten finden sich:

- (Halbautomatische) Ermittlung von Netzwerken (z. B. aus Kommunikationsdaten oder aus gemeinsam benutzten oder bearbeiteten Ressourcen),

- Nutzung der Netzwerke zur Expertensuche und auch zum (automatischen) Empfehlen von möglichen Kommunikationspartnern/Experten,

- Unterstützung von (Online-)Communitys und anderen Möglichkeiten des Austausches zu einem gemeinsamen Kontext (z. B. Foren),

- Awareness-Funktionen in der Form von impliziten Hinweisen auf Veränderungen oder aktuelle Ereignisse im persönlichen Netzwerk, z. B. eine neue berufliche Situation eines Kontaktes.
Die Leistung bzw. der Vorteil der Dienste besteht darin, eine Übersicht über die vorhandenen Kanten bereitzustellen und jedes Individuum stets mit den aktuellsten Informationen zu den mit ihm verknüpften Knoten zu versorgen. Gleichzeitig soll auch die Bildung neuer Kanten unterstützt werden. Es wird möglich, eigene Qualitäten einem größeren Publikum darzustellen, dabei mehr Informationen in kürzerer Zeit auszutauschen und schneller einen gemeinsamen Kontext für tiefer gehende Konversationen zu starten (Auswahl der Aspekte nach [TeAl05]).

Die Verbreitung von Social-Networking-Diensten ging von einer Reihe von Angeboten aus, die frei im World Wide Web nutzbar sind. Die derzeit bekanntesten Anwendungen richten sich an Jugendliche und Studierende (z. B. http://www. myspace.com, http://www.facebook.com, http://www.studivz.de). Social-Networking-Dienste sind jedoch auch für eine Vielzahl von Bereichen des täglichen Lebens zu finden, z. B. zu Zwecken

- des Datings/der Online-Partnersuche (z. B. http://www.friendster.com),

- des Austausches zu gemeinsamen Interessen und Hobbies (z. B. http://www. netzathleten.de) und

- der Koordination von politischem Handeln bzw. der Mobilisierung von Wählern bzw. Wahlkampfspenden (z. B. http://my.barackobama.com).

Darüber hinaus sind verschiedene Dienste speziell auf geschäftliche Kontakte bzw. berufstätige Personen ausgerichtet (z. B. http://www.linkedin.com, http://www. xing.de). Die Motivation zur Nutzung dieser Dienste liegt vor allem in der Möglichkeit, geschäftliche Kontakte zu verwalten, also bereits bestehende (Geschäfts-)Beziehungen zu pflegen oder neue Geschäftspartner zu finden und dabei auch gleich einen gemeinsamen Kontext (z. B. zur Unterstützung des Aufbaus von Vertrauen) $\mathrm{zu}$ finden. Der Funktionsumfang solcher Dienste (z. B. in Hinblick auf die Suchmöglichkeiten oder die Vermittlung von Kontakten) ist in der Regel deutlich größer als bei Anwendungen aus dem privat-persönlichen Kontext.

\section{Status quo: Social-Networking-Dienste in Unternehmen}

Nachdem der vorherige Abschnitt den Hintergrund und die Motivation für den Einsatz von Diensten als Grundlage für die 
technische Abbildung von Sozialen Netwerken gegeben hat, wird dieses Kapitel den Entwicklungsstand von Social-Networking-Diensten im Unternehmenseinsatz aufzeigen. Zunächst wird im Speziellen auf Anwendungen zur Expertensuche und zum Expertisemanagement eingegangen. Anschließend wird nach offenen und geschlossenen Social-Networking-Diensten unterschieden und es werden jeweils mehrere Anwendungsbeispiele vorgestellt.

\subsection{Expertisemanagement und Gelbe Seiten}

Ein wichtiger Grund für den Einsatz von Social-Networking-Diensten in Unternehmen ist das Finden von Experten zu bestimmten Themen, um die Mitarbeiter bei der Lösung i.d. R. komplexer Aufgaben $\mathrm{zu}$ unterstützen. Hintergrund der Notwendigkeit ist der Umstand, dass fehlende Transparenz von Mitarbeiterkompetenzen in mitarbeiterstarken oder verteilten Organisationen zu einem Problem werden kann, nachdem das Suchen nach zuständigen bzw. qualifizierten Personen Zeit und Geld kostet. Die ursprüngliche Lösung für diese Problematik stellen Gelben-Seiten-Anwendungen dar. Diese wurden meist als Ergänzung eines Internet-basierten Telefonbuchs um zusätzliche Einträge zur Expertise oder Aufgabe der eingetragenen Mitarbeiter realisiert.

Solche Expertenverzeichnisse stellen die Unternehmen vor verschiedene Herausforderungen. Das Hauptproblem ist die Erfassung der jeweils aktuellen Information zur Expertise, bzw. der Aufwand, der für die Aktualisierung der Informationen in den Profilen zu erbringen ist. In den einzelnen Systemen werden hierfür verschiedene Lösungen eingesetzt: Ein Ansatz ist die Integration mit anderen Anwendungen im Unternehmen (z. B. Kommunikationssoftware, Identitätsmanagement, Human-Resources-Systemen). Eine zweite Lösungsmöglichkeit stellt der häufig anzutreffende Versuch dar, Informationen über die Expertise eines Mitarbeiters automatisch aus den Dokumenten zu extrahieren, die dieser Mitarbeiter erstellt.

Hierzu gibt es bereits eine breite Basis an aktuellen Forschungsarbeiten. Eine der ersten Arbeiten im Bereich Expertenfindung ist das System Answer Garden [Acke94; AcMc96]. Von denselben Autoren stammen auch verschiedene Fallstudien zu Expertise und Expertensuche [McAc98]. Aktuelle Arbeiten im Bereich des Expertisemanagement bzw. der Expertensuche sind bei [AcPW03; BeRW04; ReWi06; Reic06] zu finden.

Unberücksichtigt bei Gelbe-Seiten-Anwendungen bleiben die Web-2.0-Konzepte der aktiven Benutzerbeteiligung bei der Bereitstellung der Daten sowie die explizite Nutzung sozialer Netzwerke zur Erleichterung der Herstellung eines gemeinsamen Kontextes, z. B. die Nutzbarmachung der Kenntnis von sozialen Beziehungen zwischen Benutzern bei der Suche oder Bestätigung von Expertise.

\subsection{Offene Social-Networking- Dienste}

Social-Networking-Dienste ergänzen reine Gelben-Seiten-Anwendungen um diese beiden wichtigen Web-2.0-Konzepte. Sie bieten den Benutzern die Möglichkeit, ihre persönlichen Kontakt- und Expertisedaten selbst zu aktualisieren. Zusätzlich kann jeder Nutzer sein Netzwerk, d. h. Kontakte zu anderen Nutzern, mit Hilfe von SocialNetworking-Diensten verwalten. Der Vorteil ist dabei, dass soziale Netzwerke explizit dargestellt und für andere Nutzer offensichtlich werden. Somit wird die Beziehungsanbahnung vereinfacht und die aus der Theorie der "strength of weak ties“ resultierenden Vorteile können besser genutzt werden. Die Erfahrung mit den Anwendungen zeigt dabei, dass neben dem direkten Nutzen durch diese Zusatzinformation häufig auch eine größere Motivation der Benutzer erreicht wird, selbst $\mathrm{Da}$ ten beizutragen.

Als offene Social-Networking-Dienste werden im Folgenden alle solchen verstanden, die keinen, oder nur sehr rudimentären Zugangsbeschränkungen unterliegen und allen Nutzern im WWW offen stehen. Die im deutschsprachigen Raum bekanntesten offenen Dienste für professionelle Nutzung sind Xing und LinkedIn.

Xing wurde im August 2004 unter dem Namen OpenBC gestartet, zählt inzwischen über zwei Millionen Mitglieder und möchte primär das IT-gestützte Knüpfen und Pflegen geschäftlicher Kontakte unterstützen [Renz07]. Die Funktionsweise des Dienstes ist relativ simpel: Jeder Nutzer erstellt ein eigenes Profil inklusive seiner Kontaktdaten, seines bisherigen beruflichen Werdegangs und seiner beruflichen (und privaten) Interessen (vgl. Bild 2 links). Durch die Möglichkeit, die meisten Informationen individuell (für einzelne Nutzer oder Nutzergruppen) freizugeben, wird auch die Pflege der Privatsphäre als Teil des Identitätsmanagements unterstützt.
Das Anlegen eines Profils und einfache Suchfunktionen sind im Rahmen einer kostenlosen Mitgliedschaft möglich. Dabei werden nicht nur die Profile der gefundenen Benutzer angezeigt, sondern auch die Verbindung, die man zu diesen Benutzern im sozialen Netzwerk hat, d. h. über welche anderen Benutzer man die gefunden Benutzer kennt. Die Nutzung spezifischer Suchfunktionen nach potentiellen Geschäftspartnern oder Mitarbeitern, aber auch nach ehemaligen Kollegen (Alumni) ist dann aber nur über eine kostenpflichtige Premium-Mitgliedschaft möglich. Zusätzlich zur Suche und zur Profilverwaltung bietet Xing noch das Einrichten von so genannten Gruppen (oder Communitys), in denen sich Experten in Foren austauschen können.

Eine besondere Rolle kommt bei Xing schließlich der eigenen Startseite zu, die als Schaltzentrale für die Nutzung gesehen werden kann und sich weitestgehend an die persönlichen Bedürfnisse anpassen lässt [Lamp07, $131 \mathrm{ff}$.$] . Auf der Startseite findet$ man beispielsweise eine Übersicht über neue Mitglieder des Netzwerks, über die letzten Besucher des eigenen Profils, über die nächsten Geburtstage seiner Kontakte, über öffentliche Termine in der eigenen Region und über neue Artikel in diversen themenbezogenen Gruppen. Sie liefert damit einen wesentlichen Beitrag zur Awareness, also dem Bewußtsein der Einzelnen über die Kenntnisse und Aktivitäten der anderen.

LinkedIn wurde bereits 2002 als Business-Plattform gegründet und ist mit über elf Millionen Nutzern die weltweit größte ihrer Art. Auch hier erstellt der Nutzer zunächst ein eigenes Profil und kann dann unter anderem auf eine umfangreiche Jobbörse, eine Plattform für Dienstleistungsanbieter, eine Frage- und Antwortplattform sowie auf zahlreiche unterschiedliche Foren und Gruppen zugreifen. Bereits die für das Profil abgefragten Informationen und angebotenen Funktionen unterscheiden sich jedoch wesentlich von Xing. Auf den ersten Blick (vgl. Bild 2 rechts) fällt auf, dass auf ein Foto verzichtet wird, wie in Amerika auch bei Bewerbungen üblich. Der Fokus liegt nicht auf der Kontaktbzw. Profilverwaltung, sondern auf der Anbahnung von Geschäftsbeziehungen. In diesem Zusammenhang ist als zentrales Element von LinkedIn der Empfehlungsalgorithmus zu nennen, welcher den Nutzern anhand von Data-Mining-Verfahren andere Nutzer empfiehlt.

Für ein Unternehmen bieten die beiden Dienste die Möglichkeit, dass sich darüber 


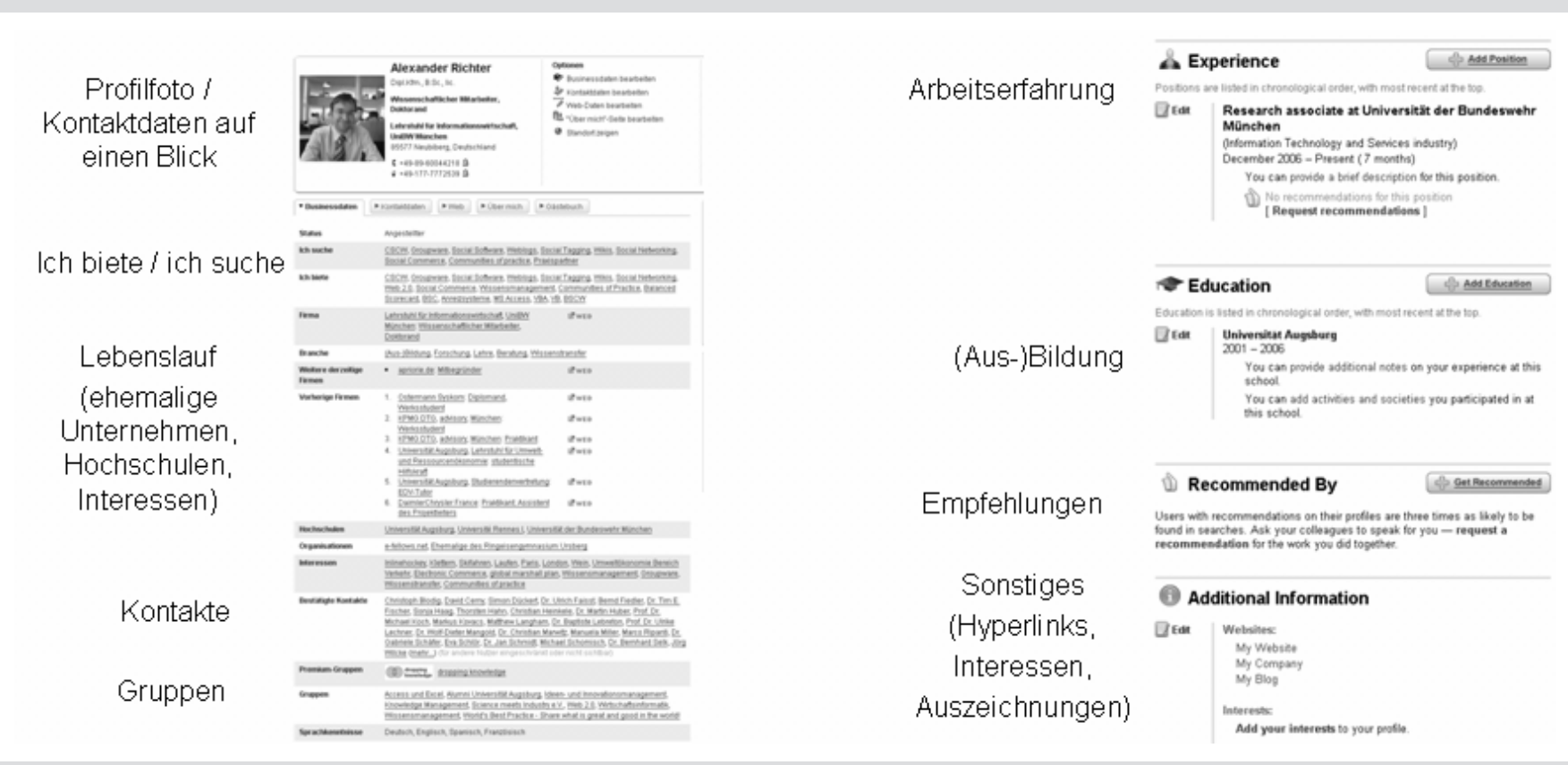

Bild 2 Die Social-Networking-Dienste Xing (links) und Linkedln (rechts)

die eigenen Mitarbeiter untereinander und mit anderen Unternehmen besser vernetzen. Durch die öffentliche Zugänglichkeit und kostenlose Grundverfügbarkeit finden diese Dienste auch ohne explizite Werbung in Unternehmen bereits regen Zulauf bei den Mitarbeitern. Einer der Hauptgründe, sich in einem offenen Social-NetworkingDienst zu präsentieren, ist dabei heute zwar meist der Wunsch, Kontakte zu alten Freunden, Kommilitonen oder ehemaligen Kollegen aufrecht zu erhalten. Darüber hinaus finden sich aber auch immer mehr Möglichkeiten für die berufliche Nutzung. Einträge in offenen Social-NetworkingDiensten sind in manchen Unternehmen sogar explizit erwünscht. Das Unternehmen hat damit die Möglichkeit, seine Expertenkenntnisse einer breiteren Öffentlichkeit darzustellen oder das Finden interner Experten zu ermöglichen, ohne eine eigene Plattform einzuführen. Insbesondere wenn die Einbeziehung von ehemaligen Mitarbeitern interessant oder wichtig ist, findet dieses Vorgehen große Verbreitung. So hat IBM erst kürzlich mit der Xing-Gruppe „The greater IBM connection“ einen Piloten zur Vernetzung von aktuellen Mitarbeitern und Alumni gestartet. IBM motiviert Mitarbeiter und Alumni dabei beispielsweise mit der Finanzierung einer Premium-Mitgliedschaft bei Xing.

\subsection{Geschlossene Social-Networking-Dienste}

Während die oben beschriebenen offenen Dienste sowohl der Expertensuche und Kontaktanbahnung und -pflege in sowie zwischen verschiedenen Unternehmen dienen, sind geschlossene Dienste nur innerhalb der Unternehmensintranets - und damit nur für die Mitarbeiter eines Unternehmens - zugreifbar. Die Dienste bieten damit die Möglichkeit, mehr unternehmensinterne Daten zu den Mitarbeitern bereitzustellen und diese Daten auch teilweise automatisch aus firmeninternen Systemen einzuspeisen.

Einer der bisher am weitesten ins Unternehmen integrierten Dienste zur Expertensuche stellen die IBM Blue Pages (BP) mit aktuell über 475.000 Profilen dar. Das besondere daran ist die Integration ins Unternehmen und die restliche UnternehmensIT als eine zentrale Datenbasis für (unternehmensöffentliche) Mitarbeiterinformation. So werden die BP bspw. direkt aus der zentralen HR-Datenbank gespeist. Insgesamt sind die BP heute mit über fünfzig Anwendungen vernetzt, die Daten einspeisen oder aus den BP beziehen. So laufen alle Anwendungsauthentifizierungen innerhalb des IBM-Intranets über die BP - die $\mathrm{BP}$ sind also die zentrale Identitätsmanage- mentanwendung bei IBM. Neben Telefonnummern, E-Mail-Adressen, InstantMessaging-Erreichbarkeiten und der hierarchische Einordnung des Mitarbeiters in Form der Berichtslinie haben die Mitarbeiter verschiedene Möglichkeiten zur Selbstdarstellung. Über die „My Blue Pages List“ ist weiterhin ein einfaches Erfassen und Verwalten von Kontakten und damit von sozialen Netzwerken möglich.

Als zentrale Anwendung zum Zugriff auf Kontaktinformation im Unternehmen werden über die BP über 3,5 Mio. Suchanfragen pro Woche abgewickelt. Als reine Zeitersparnis gegenüber klassischen Telefonbuch-Lösungen für die Mitarbeiter rechnet IBM für die BP 72 Minuten pro Mitarbeiter und Monat [Schü07].

Aufbauend auf der oben beschriebenen Basis werden die BP aktuell systematisch um Web-2.0-Funktionalitäten ergänzt. Insbesondere ist es damit möglich, sich selbst und Kollegen zu „taggen“, d. h. jeder kann sich und anderen frei wählbare Schlagworte z. B. zur Expertise zuordnen. Dadurch wird die Aufgabe der Erfassung einer jeweils aktuellen Expertiselandkarte auf die Schultern aller Mitarbeiter gelegt [FaLa06]. Weiterhin ermöglichen die zukünftigen BP die graphische Darstellung des persönlichen Kontaktnetzwerks (thematisch und geographisch), wodurch in- 
nerhalb der IBM das „Business Networking" erleichtert werden soll.

Einen Großteil dieser Eigenentwicklungen bietet IBM inzwischen auch Kunden an, in Form des Moduls Profiles im neuen Social-Software-Paket Lotus Connections (vgl. Bild 3). Darin werden verschiedene Funktionalitäten verknüpft:

- Jeder Mitarbeiter hat die Möglichkeit sein eigenes Profil zu erstellen und zu editieren

- Seinem Profil können von ihm Tags zugeordnet werden, wie z. B. die Namen aktueller Projekte oder seine Fachkenntnis. Eine Zuordnung von Tags durch Kollegen wie in den BP ist momentan noch nicht möglich.

- Jedes Profil kann zusätzlich mehreren Communitys oder Teams zugeordnet sein. Dadurch wird es möglich, Mit- arbeiter mit ähnlichen Interessen oder Hintergründen zu finden.

- Außerdem können automatisch Informationen aus anderen Anwendungen (Blogs, Social Bookmarking) in das Profil integriert werden. Blogs, Social Bookmarks und Activities werden outof-the-box automatisch ins Profil integriert. Weitere Datenquellen können durch den Administrator definiert werden.

Auf der Basis dieser Funktionen aufsetzend soll es jedem Mitarbeiter ermöglicht werden, so schnell, präzise und leicht wie möglich einen passenden Ansprechpartner für die Lösung eines Problems zu finden. Durch die Integration mit Lotus Sametime kann auch die unternehmensinterne Kommunikation (über Instant Messaging und/ oder Voice over IP) unterstützt werden.
Neben Profiles beinhaltet Lotus Connections noch vier weitere Module: Communities, welches Leute mit ähnlichen Interessen zusammen bringen sollen, Activities zur Teamkoordination, Bookmarks zur gemeinsamen Verwaltung von Internet-Lesezeichen und Blogs.

Vergleichbare Funktionalitäten bieten die drei Produkte aus der Produktkategorie Aqualogic des Enterprise-Software-Anbieters BEA. Neben den Komponenten „Ensemble“ (erlaubt z. B. die Benutzung von Mash-Ups) und Pages (erzeugt Enterprise-Content auf Basis von z. B. Wikis oder Blogs) ist die Komponente PathWays integriert. Diese verfolgt das Ziel, Informationen und Expertenwissen im Unternehmen leichter und schneller verfügbar zu machen.

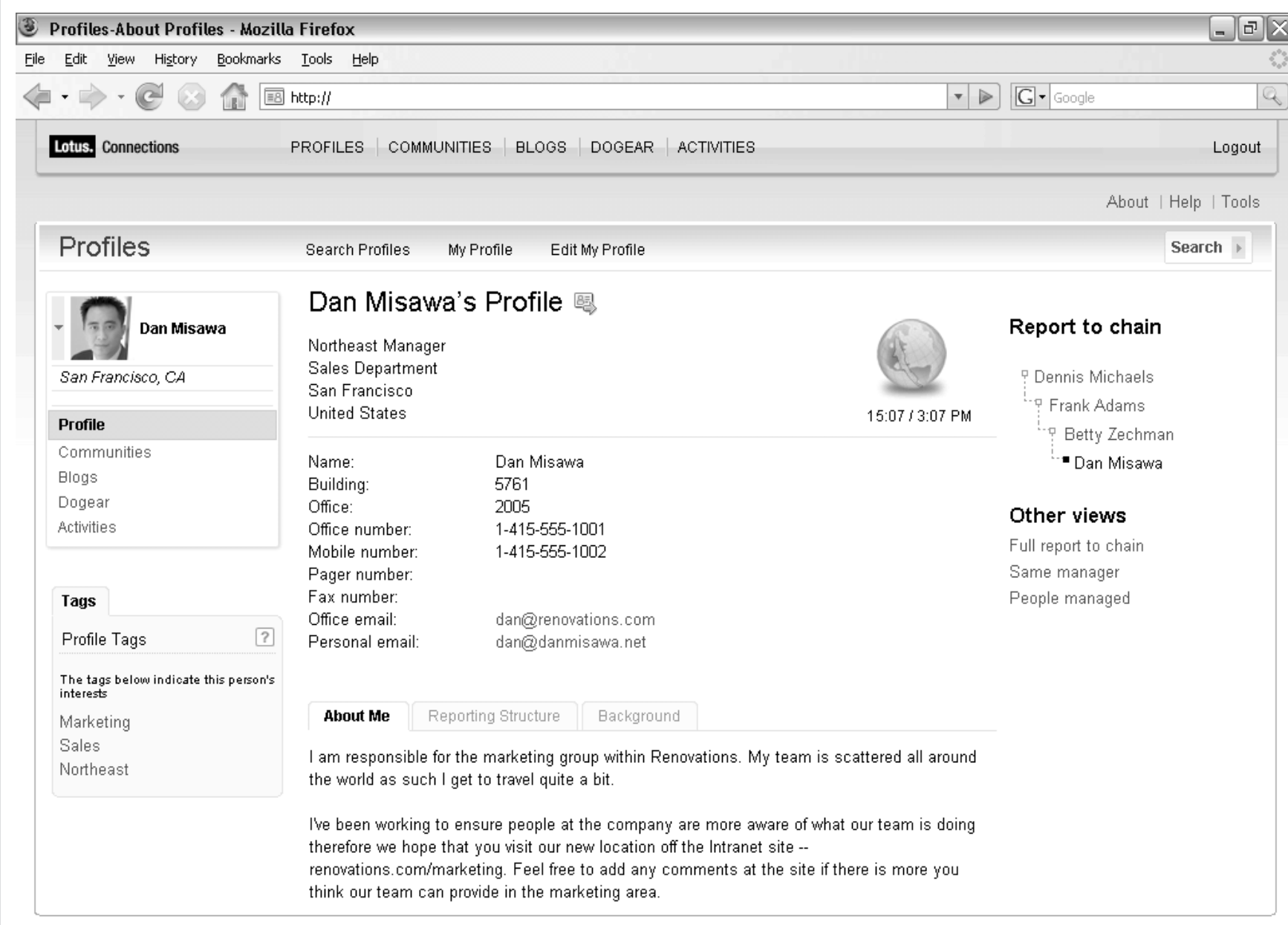

Bild 3 Profildarstellung in IBM Connections Profiles 
Ein zentraler Unterschied $\mathrm{zu}$ den vorgestellten IBM-Anwendungen liegt im Ausgangspunkt für die Entwicklung: Während IBM Lotus Connections/Profiles eher personenzentriert arbeitet, ist BEA Pathways mehr inhaltezentriert. Das bedeutet, dass sich Personen mehr durch die von ihnen erstellten und gespeicherten Inhalte definieren. Um das Auffinden von Informationen zu erleichtern, kommt bei BEA ein Algorithmus namens "Activity-Rank“ zum Einsatz. Dieser ordnet Suchergebnisse für eine bestimmte Anfrage anhand folgender Kriterien: So werden die Beziehung zwischen dem suchenden Nutzer und anderen Nutzern, die ein bestimmtes Dokument verwenden, dem Dokument zugewiesene Tags, dem Autor des Dokuments und der Benutzungshäufigkeit des Dokuments als Suchkriterien verwendet. Für das Auffinden von Experten werden die eigenen Kontakte sowie Tags, die anderen Benutzern zugewiesen wurden, verwendet.

Ähnliche Angebote finden sich auch bei anderen Anbietern. Das Spektrum reicht hier von leicht erweiterten Webbasierten Telefonbuchanwendungen bis hin zu Social-Networking-Anwendungen, die mit weiteren Social-Software-Typen verknüpft sind. Auch Microsoft steuert seit Februar 2007 mit dem Sharepoint-ServerAdd-On „Knowledge Network“ eine Lösung zu diesem Bereich bei. Mit elgg (http://www.elgg.org) gibt es sogar schon ein Open-Source-Produkt, das den Vergleich mit den kommerziellen Lösungen nicht scheuen muss.

\section{Fazit und Ausblick auf notwendige Entwicklungen}

Social-Networking-Dienste haben großes Potenzial, die softwaregestützte Anbahnung der Zusammenarbeit in den Unternehmen $\mathrm{zu}$ unterstützen. Dies wird erreicht, indem sie Funktionen zum Beziehungs-, Expertise- und Identitätsmanagement zur Verfügung stellen. Sie erleichtern so das Finden von Experten und stellen die für die Erlangung eines gemeinsamen Kontextes nötigen Informationen zur Verfügung. So kann die Kommunikation zwischen den Mitarbeitern wesentlich effizienter und vor allem flexibler gestaltet und damit neue Organisations- und Kommunikationsstrukturen ermöglicht werden. Mit der Verbesserung der Kommunikation einher geht die effektivere Nutzung und Vernetzung des Wissens aller Mitarbeiter und eine damit verbundene bessere Ausschöpfung des Humankapitals. Die Mitarbeiter werden auch nach der Zusammenarbeit darin unterstützt, ihre Kontakte aufrecht zu erhalten und aktuelle Informationen über Entwicklungen ihrer Kollegen zu bekommen.

Derzeit werden in den Unternehmen meist einfache Gelbe-Seiten-Anwendungen eingesetzt. Diese bieten ihren Nutzern keinerlei Zusatzinformation zu Netzwerken oder zu sonstiger Meta-Information. Social-Networking-Dienste gehen hier einen Schritt weiter. Durch die dezentrale Pflege der Profile, verstärkte Integration mit anderen Diensten im Unternehmen und die Berücksichtigung von sozialen Netzwerken kann hier eine ganz neue Nutzungsqualität erreicht werden. Zur Aufwandsminimierung und Nutzenerhöhung beitragen können zusätzlich Web-2.0-Ideen und Konzepte wie einfaches Tagging von Mitgliedern aus unterschiedlichen Perspektiven oder die Visualisierung der Expertise über Tag Clouds, die aus Dokumenten oder anderen Beiträgen der Benutzer (z. B. in Wikis) gewonnen worden sind [FaLa06].

Die wichtigste aktuelle Entwicklung im Bereich der Social-Networking-Dienste ist die Integration - z. B. mit anderen SocialNetworking-Diensten und anderen Diensten im Unternehmen. Hierzu gibt es verschiedene Aktivitäten:

Die Kopplung mit dem Identitätsmanagement des Unternehmens [siehe hierzu auch KoMö05] und anderen Diensten des Unternehmens (insbes. HR-Systemen) ist bei kommerzieller Software zum Aufbau von internen Social-Networking-Diensten wie Lotus Connections schon sehr weit verbreitet. Noch nicht so weit verbreitet ist die Möglichkeit, Profilinformation dienstübergreifend zu nutzen. So stellt beispielsweise Ning (http://www.ning.com) eine Middleware zur Verfügung, auf der SocialNetworking-Dienste (z. B. für Konferenzen) aufgebaut werden können. Diese sind dann in der Lage, Profile aus anderen Diensten zu verwenden, welche sich auf dieselbe Middleware stützen. Andere Ansätze verfolgen im Rahmen von föderiertem Identitätsmanagement die Idee der verteilten Speicherung von Profilinformation (z. B. FOAF, http://www.foaf-project.org, oder OpenID, http://www.openid.org). Leider bieten die großen offenen SocialNetworking-Dienste bisher noch keine solchen Export- oder Föderierungsmöglichkeiten. Dies wäre längerfristig aber auf jeden Fall wünschenswert. Eine solche Trennung von Daten und Benutzungsschnittstelle entspräche auch viel mehr den Paradigmen des Web 2.0. Ebenso wünschenswert wäre ein Austausch bzw. eine Föderation zwischen firmeninternen und -externen Diensten - natürlich mit geeigneten Filtern, um Datenschutz und Datensicherheit zu garantieren. All dies könnte sehr zur Aufwandsminimierung bei den Benutzern beitragen und so entscheidende Impulse zur Nutzung geben. Dies gilt insbesondere für Umgebungen, in denen Unternehmen sowohl die Nutzung interner Dienste als auch externer Dienste fördern.

Eine weitere Möglichkeit der Integration ist Nutzung von Social-Networking-Diensten in Unternehmensportalen (über Portlets, RSS-Feeds oder Webservices). Neben der Bereitstellung der dafür notwendigen offenen Schnittstellen gibt es inzwischen

\section{Abstract}

\section{Services and Applications for IT-Supported Social Networking in Companies}

The use of Social Networking Services for private purposes has been increasing considerably in the past few years. In the same time companies started to experiment with such services to support formal and informal communication and networking among their employees.

This article gives an overview of services and application for IT-supported Social Networking in companies. First, the authors place Social Networking Services in the context of Web 2.0 and Social Software. Thereupon, they discuss the benefits and the motivation of the deployment of Social Networking Software in companies, and introduce some specific IT solutions. Finally, they also address necessary future developments in the field of Social Networking Services.

Keywords: Social Networking Service, Expert Finding, Yellow Pages, Knowledge Management, Web 2.0, Social Software, Enterprise 2.0 
bei verschiedenen Diensten erste Ansätze, die Plattformen selbst sehr weit anpassen oder erweitern zu können. Einen beachtlichen Schritt hat hier das einstige Studentennetzwerk Facebook unternommen, als es im Mai 2007 die Plattform öffnete und anderen Firmen erlaubte, ihre Programme in Facebook zu integrieren. Die Möglichkeiten, die sich durch die Öffnung des Netzwerks nun den Benutzern bieten, lassen sich zum Zeitpunkt der Fertigstellung dieses Beitrags nur erahnen.

\section{Literatur}

[Acke94] Ackerman, Mark: Augmenting the organizational memory: a field study of answer garden. In: Proceedings International Conference on Computer-Supported Cooperative Work, Chapel Hill, NC. New York 1994, S. 243-252.

[AcMc96] Ackerman, Mark; McDonald, David W. Answer Garden 2: Merging Organizationa Memory with Collaborative Help. In: Proceedings International Conference on ComputerSupported Cooperative Work, Boston, MA New York 1996, S. 97-105.

[AcPW03] Ackermann, Mark; Pipek, Volkmar; Wulf, Volker (Hrsg.): Sharing Expertise - Beyond Knowledge Management. MIT Press, Cambridge 2003.

[Alby06] Alby, Tom: Web 2.0 - Konzepte, Anwendungen, Technologie, Hanser Verlag, Hamburg 2006.

[BaBo03] Barabasi, Albert-Laszio; Bonabeau, Eric: Scale-Free Networks. In: Scientific American (2003) May, S. 50-59.

[BeRW04] Becks, Andreas; Reichling, Tim; Wulf, Volker: Expertise Finding: Approaches to Foster Social Capital. In: Huysman, Marleen; Wulf, Volker (Hrsg.): Social Capital and Information Technology. MIT Press, Cambridge 2004, S. 333-354.

[BuMa07] Bughin, Jacques; Manyika, James: How businesses are using Web 2.0: A McKinsey Global Survey. http://www.mckinseyquarterly.com/ article_page.aspx?ar=1913, 2007, Abruf am 2007-05-15.

[Cast03] Castells, Manue: Das Informationszeitalter 1. Der Aufstieg der Netzwerkgesellschaft.
Studienausgabe. Wirtschaft - Gesellschaft - Kultur. Öeske + Budrich, Opladen 2003

[CrLW05] Cross, Rob; Liedtka, Jeanne; Weiss, Leigh: A Practical Guide to Social Networks. In: Harvard Business Review (2005) 3, S. 124132.

[EhCa05] Ehrlich, Karin; Carboni, Ingrid: Inside Social Network Analysis. Technical Report, IBM Watson Research Center,

http://domino.watson.ibm.com/cambridge/ research.nsf/

58bac2a2a6b05a1285256b30005b3953/

3f23b2d424be0da6852570a500709975?

OpenDocument, 2005, Abruf am 2007-06-05.

[FaLa06] Farrell, Stephen; Lau, Tessa: Fringe Contacts: People-Tagging for the Enterprise. Technical Report, IBM Almaden Research Center, http://domino.watson.ibm.com/library/ CyberDig.nsf/

7d11afdf5c7cda94852566de006b4127/

53299 b30ad986c78852571b0004f46a9?

OpenDocument, 2006, Abruf am 2007-06-20.

[Furn97] Furnham, Adrian: The psychology of behaviour at work: the individual in the organization. Taylor \& Francis, Hove 1997.

[Glad00] Gladwell, Malcom: The Tipping Point How Little Things Can Make a Big Difference. Little, Brown and Company, Boston 2000.

[Gran73] Granovetter, Mark: The Strength of Weak Ties. American Journal of Sociology 78 (1973) 6, S. 1360-1380.

[HiWi05] Hippner, Hajo; Wilde, Thomas: Social Software. In: Wirtschaftsinformatik 47 (2005) 6, S. 441-444.

[HeSK06] Hein, Oliver; Schwind, Michael; König, Wolfgang: Scale-Free Networks - The Impact of Fat Tailed Degree Distribution on Diffusion and Communication Processes. In: Wirtschaftsinformatik 48 (2006) 4, S. 267-275.

[KoMö05] Koch, Michael; Möslein Katrin: Identities Management for E-Commerce and Collaboration Applications. In: International Journal of Electronic Commerce (IJEC) 9 (2005) 3, S. 11-29.

[Lamp07] Lamprecht, Stephan: Xing - Das Buch Netzwerken leicht gemacht. Heise, o. O. 2007.

[McAf06] McAfee, Andrew: Enterprise 2.0 - The Dawn of Emergent Collaboration. In: MITSloan Management Review 47 (2006) 3, S. 21-28.

[McAc98] McDonald, David. W.; Ackerman, Mark: Just Talk to Me: A Field Study of Expertise Location. In: Proceedings International Conference on Computer-Supported Cooperative Work, Seattle, WA. New York 1998, S. 315-324.
[Milg67] Milgram, Stanley: The Small Word Problem. In: Psychology Today (1967) 1, S. 62-67.

[More34] Moreno, Jacob. L.: Who Shall Survive? Nervous and Mental Disease Publishing Company, Washington 1934.

[NaWS02] Nardi, Bonnie. A.; Whittaker, Steve; Schwarz, Heinrich: NetWORKers and their Activity in Intensional Networks. In: Computer Supported Collaborative Work 11 (2002) 1-2, S. 205-242.

[ORei05] O'Reilly, Tim: What is Web 2.0 - Design Patterns and Business Models for the Next Generation of Software.

http://www.oreillynet.com/pub/a/oreilly/tim/ news/2005/09/30/what-is-web-20.html, 2005, Abruf am 2007-06-12.

[Reic06] Reichling, Tim: ExpertFinding: Auffinden von Experten in großen Organisationen. In: Heinecke, A. M.; Paul, H. (Hrsg): Proceedings Mensch \& Computer 2006: Mensch und Computer im Strukturwandel. München 2006, S. 361-366.

[ReWi06] Reichling, Tim; Wirth, Matthias: Yellow Pages in einem Verband - Experten finden und zusammenführen. In: VDMA, Journal Arbeit 6 (2006) 1, S. 22-23.

[Renz07] Renz, Florian: Praktiken des Social Networking. Eine kommunikationssoziologische Studie zum online-basierten Netzwerken am Beispiel von openBC (XING). Hülsbusch, Boizenburg 2007.

[RiKo07] Richter Alexander; Koch Michael: Social Software - Status quo und Zukunft. Technischer Bericht Nr. 2007-01, Fakultät für Informatik, Universität der Bundeswehr München 2007.

[Sche00] Scheler, Uwe: Erfolgsfaktor Networking. Campus Sachbuch, Frankfurt/Main 2000.

[Schü07] Schütt, Peter: mITeinander im Web 2.0. Vortrag auf dem IBM Symposium 2007, Zürich, 2007-05-10.

[TeAl05] Teten, David; Allen, Scott: The virtual handshake: opening doors and closing deals online. McGraw-Hill, New York 2005

[Well97] Wellman, Barry: An Electronic Group is virtually a Social Network. In: Sara Kiesler (Hrsg): Culture of the Internet. Lawrence Erlbaum Associates, Hillsdale 1997, S. 179-205.

[Well01] Wellman, Barry: Computer networks as social networks. In: Science 293 (2001), S. 20312034

[Weng98] Wenger, Etienne: Communities of Practice - Learning, Meaning and Identity. University Press, Cambridge 1998. 